\title{
Malarial Parasite Identification Using Convolution Neural Network
}

\author{
S.Kavitha ${ }^{1}$, S. Sathyavathi ${ }^{2}$, R. Priyadharshini ${ }^{3}$ and S.Varshini ${ }^{4}$ \\ ${ }^{1}$ Assistant Professor, Department of Information Technology, \\ Kumaraguru College of Technology, Coimbatore, India. \\ ${ }^{2}$ Assistant Professor, Department of Information Technology, \\ Kumaraguru College of Technology, Coimbatore, India. \\ ${ }^{3,4}$ Student, Department of Information Technology, Kumaraguru College of Technology. Coimbatore, India.
}

\section{ABSTRACT}

Malaria - a dreadful and deadly disease caused by a parasite belong to the plasmodium family that commonly infects a female Anopheles mosquito which bite on humans. With the symptoms, the disease can be diagnosed by trained lab technicians who will examine the microscopic blood smear images. Developing an automatic, accurate and efficient model for detecting this disease will reduce the requirement for the trained human resource and it will improve the diagnosis efficiency. Deep learning neural networks can be used to improve the efficiency and the accuracy of the diagnosis. In this paper, we propose a model using Convolutional Neural Network (CNN) for the examination of malaria from the microscopic human red blood smear images. This model will provide a rapid, accurate, low cost outcome. Our model differentiates the infected and uninfected cell images by training the convolutional neural networks. The algorithm involves the methods and architectures of computer vision, image processing operations and deep learning. The proposed CNN model can examine the malarial parasites from microscopic images with an accuracy of 68.38\%, in 10000 checkpoint operations.

KEY WORDS: BLOOD SMEAR, CNN, DEEP LEARNING, MICROSCOPIC, PLASMODIUM PARASITES.

\section{INTRODUCTION}

Malaria is infectious and deadly disease. It is caused by the Plasmodium parasites. Malaria- mosquito-borne disease are spread by the bites of the infected female Anopheles mosquitoes. Out of many only 5 Plasmodium species cause malaria in human. They are Plasmodium falciparum, Plasmodium vivax, Plasmodium malariae, Plasmodium ovale, and Plasmodium knowlesi. If an

\section{ARTICLE INFORMATION}

*Corresponding Author: sathyavathi.s.it@kct.ac.in Received 4th Oct 2020 Accepted after revision 10th Dec 2020 Print ISSN: 0974-6455 Online ISSN: 2321-4007 CODEN: BBRCBA

Thomson Reuters ISI Web of Science Clarivate Analytics USA and Crossref Indexed Journal

\section{Clarivate
Analytics}

NAAS Journal Score 2020 (4.31) SJIF: 2020 (7.728)

A Society of Science and Nature Publication,

Bhopal India 2020. All rights reserved.

Online Contents Available at: http//www.bbrc.in/

Doi: http://dx.doi.org/10.21786/bbrc/13.11/12 infected female Anopheles mosquito bit a person, parasites in the mosquito emerge into human blood of the person and start destructing the oxygen-carrying human red blood cells (RBC). The symptoms are visibly noted in a few days or a week after the mosquito bites. Initial symptom is with the fever.

Without causing any symptoms the parasites can live in the human body over a year also. The delay in treatment for malaria can lead to multiple complications in the human body or sometimes it leads to death. Early detection of this dreadful disease can save life of the human. World Health Organisation (WHO) confirm that the diagnosis of the disease involves careful testing of the blood smear at 100X magnification. Lab technicians test the blood and count how many red blood cells contain parasites for every 5,000 cells. This process is complex. 
To improve the accuracy deep learning models can be used which will save the human life.

\section{METHODOLOGY}

Publicly available malaria dataset is used to conduct the experiments. The following are the steps involved: Importing Libraries, Loading the data, Data preprocessing, Data augmentation, Plotting images and its labels to understand how does an infected cell and uninfected cell looks like, Splitting data in Train, Evaluation and Test set, Creating a Convolution Neural Network function, Wrapping it with TensorFlow Estimator function, Training the data on Train data, Evaluating on evaluation data, Predicting on Test data, Plotting the predicted image and its respective True value and predicted value.

Dataset Collection: The dataset is taken from the publicly available official Kaggle Website. Total of 27,558 cell images are taken which comprises of both parasitized and uninfected cell images. Number of parasitized and uninfected cell images are equal. Reduction of images is done by carefully by annotating the images, where we remove the falsely and suspicious images, Number of false and suspicious images are about 647. Removing that from the data set which results in 26,161 images. Out of 26161 images, parasitized data stands 13,132 and the remaining are uninfected cell images.

Figure 1: Pre-processed Image Dataset

Unifected:0 Infected:1 Infected:1 Infected:1 Infected:1 Infected:1

Data Pre-Processing: Data pre-processing is a basic step as the quality of image and the useful information that can be extracted from the image that directly affects the performance of the model to learn and predict. The behaviour and performances of the model purely depends on the data. After the image data acquisition process, most deep learning models perform various pre-processing methods.This would enhance the quality of the image by eliminating the noise, enhancing theillumination, improvising the colour variations inherent in the collection and staining phase of the image. So, data pre-processing is an important and essential process in any deep learning experiments. In this experiment, images are manually corrected. Images are resized and image patches are rescaled to map the features between 0 and 1 range as per the model requirement which leads to faster convergence. Data augmentation was applied to training data to improve the model performances. Preprocessing is done to improve the image quality and to reduce differences in the images that would avoid the complications in the forthcoming operations.

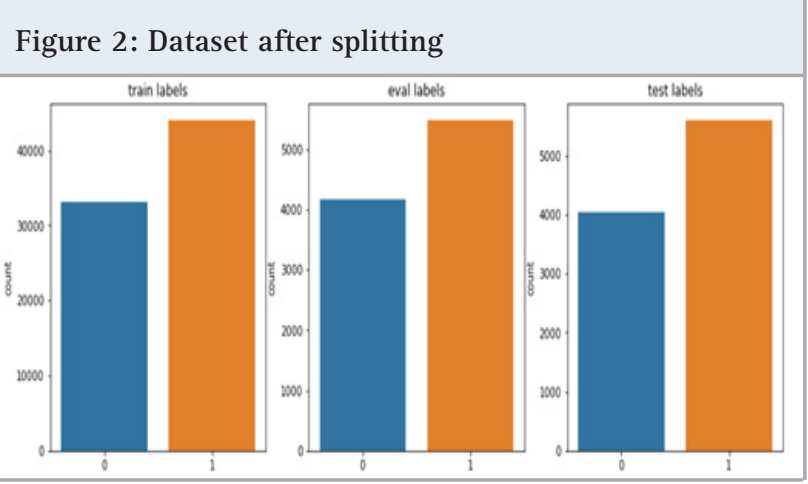

Data Splitting: Split the dataset into three sets in the ratio of 80 (training): 10 (validation): 10 (test).We used sci-kit (open source learn machine learning library and imported "train_test_split" from sklearn_model_selection which splits arrays or matrices into random train and Test subsets. The Random state ensures that the splits generated are reproducible. The provided random state is used as a seed to the random number generator. This ensures that the random elements are generated in the same order. To ensure the train-test splits are always deterministic, random state which set a seed for the random generator is used. If the seed is not set properly, train-test split will be not same at all time.

Convolutional Neural Network: Inspired by the animal visual system, CNN is an artificial neural network. CNNs can extract features compared to conventional neural networks without losing a lot of spatial input correlations. Each layer consists of neurons that contain weights and prejudices that can be learned. After feeding data into the network and minimising the loss function at the top layer, the optimal model is acquired. A filter of value 50 with padding set to the same is used for convolution over the input volume in the convolutional layer's operation. In every max pooling layers size of 2 $\mathrm{x} 2$ pool is used. The flatten layer with output neurons receive the input as the output from the final max pooling layer. Next is the two blocks of dense layer of output neurons. Drop out layer is used which discards 50\% of the input neurons in a random manner.

The activation function Rectified Linear Unit (ReLU) is used in the convolution layers. SoftMax activation is used in the final stage of classification. Its output represents the vector which shows the probability distribution of the outcomes. The loss function sparse SoftMax is used to calculate the error between the actual value and predicted output value probabilities. Gradient Descent Optimizer is used to ensure the adaptive learning process. It optimizes the biases and weights of the network. Initially the weight 
is assigned randomly, and the biases are initialised to 0 . The activation function are applied for the batch size of 100 samples.

Figure 3: Execution result

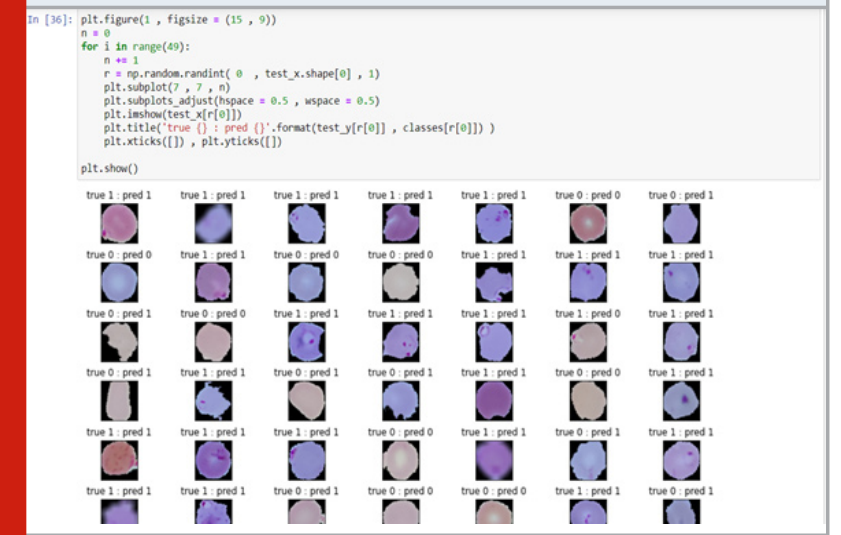

Confusion Matrix: The number of correct and incorrect predictions made by a classifier is evaluated. The summary of the predictions either correct or incorrect is given in the table named as confusion matrix. The performance of a classification model is calculated using the metrics accuracy, precision, recall, and F1-score.

\section{CONCLUSION}

In this work, we directed a progression of tests dependent on deep learning models to make efficient malarial parasite classification from classified human red blood cell smear images. We show that data augmentation methodologies used on the training set shows definite results. Our model achieves an accuracy of $68.38 \%$. In future works, the different deep learning architecture can be used and analysed to understand to improve the accuracy.

\section{Figure 4: Confusion Matrix}

In [35]: from sklearn.netrics import confusion_natrix , classification_eport, accuracy_scone

print(' \{\} $\ln \{\} \ln \{\}$ ', format(confusion natrix(test $y$, classes),

classification_eport(test $y$, classes),

$$
\text { accuracy_score(test } y \text {, classes))) }
$$

[[1970 2069]

[ 948 4659]]

precision recall f1-score support

$\begin{array}{lllll}0 & 0.68 & 0.49 & 0.57 & 4939 \\ 1 & 0.69 & 0.83 & 0.76 & 5687\end{array}$

$\begin{array}{lll}\text { accuracy } & 0.69 \quad 9646\end{array}$

\begin{tabular}{lllll} 
accuracy & & & 0.69 & 9646 \\
macro avg & 0.68 & 0.66 & 0.66 & 9646 \\
\hline
\end{tabular}

$\begin{array}{lllll}\text { weighted avg } & 0.69 & 0.69 & 0.68 & 9646\end{array}$

0.6872278664731495

\section{REFERENCES}

Confusion Matrix Guide : https://www.educative. io/edpresso/how-to-create-a-confusion-matrix-inpython-using-scikit-learn

Mahdieh Poostchi .Image analysis and machine learning for detecting malaria by Kamolrat Silamut, Richard J.Maude, Stefan Jaeger, George Thoma, Volume 194, (2018).

Muhammad Umer, Saima Sadiq Muhammad Ahmad, Saleem Ullah, Gyu Sang Choi, Arif Mehmood (2020) PP(99):1-1

Muhammad Umer. A Novel Stacked CNN for Malarial Parasite Detection by Pages 36-55.

tf.compat.v1.train.GradientDescentOptimizer Guide : https://www.tensorflow.org/api_docs/python/tf/ compat/v1/train/GradientDescentOptimizer

World Health Organization. Malaria: fact sheet. No. WHO-EM/MAC/035/E. World Health Organization. Regional Office for the Eastern Mediterranean (2014). 